\title{
Anti Chsh-Refutation of the Chsh Inequality
}

\author{
Ilija Barukčić \\ Horandstrasse, Jever, Germany \\ Email: Barukcic@t-online.de
}

Received 13 March 2016; accepted 23 April 2016; published 26 April 2016

Copyright (C) 2016 by author and Scientific Research Publishing Inc.

This work is licensed under the Creative Commons Attribution International License (CC BY). http://creativecommons.org/licenses/by/4.0/

(c) (i) Open Access

\section{Abstract}

Today, quantum-mechanical concepts i.e. such as non-locality refer to some mathematical foundations, especially to Bell's inequality and the Chsh inequality. Experimental data, analyzed while using Bell's inequality or the Chsh inequality, favor a quantum mechanical description of nature over local hidden variable theories (often referred to as local realism). In general, the use of mathematically inconsistent methods can imply a waste of money, time and effort on this account. Under some certain conditions (the assumption of independence) Bell's theorem and the Chsh inequality are already refuted. The purpose of this publication is to explore the terra incognita, the interior logic that may lie beyond Bell's original theorem and the Chsh inequality and to refute both Bell's original theorem and the Chsh inequality under any circumstances by the proof that we can derive a logical contradiction out of Bell's inequalities. Thus far, if you accept Bell's theorem or the Chsh inequality as correct, then you must accept too that $+0=+1$, which is a logical contradiction. Bell's theorem and the Chsh inequality are refuted in general. In this insight, it appears to be necessary to revisit the very foundations of quantum theory and of physics as such.

\section{Keywords}

Relativity Theory, Quantum Theory, Unified Field Theory, Causality

\section{Introduction}

In contrast to relativity theory, quantum mechanics is still not based on generally accepted principles or foundations. Theoretically, an axiomatic formalization of the mathematical foundations of quantum mechanics similar to Newton's principia is a possible approach to solve this problem but still not in sight. The core of this problem is related to Einstein's theory of relativity. In general, Einstein's special theory of relativity, supported by many experiments, requires and obeys something like a principle of locality [1] [2]. But due to Bell, "It is the requirement of locality, or more precisely that the result of a measurement on one system be unaffected by operations on a distant system with which it has interacted in the past, that creates the essential difficulty" [3]. Thus far, a 
generally accepted local realistic interpretation of quantum mechanics is still not in sight. Meanwhile, this tension between the locality of relativity theory and the non-locality of quantum mechanics is favoured by dozens of experiments performed; the philosophical implications of Bell's theorem and the physical meaning are still a matter of discussion. Especially, due to the incorrect and bold position of d'Espagnat, we are forced to accept the following:

"The doctrine that the world is made up of objects whose existence is independent of human consciousness turns out to be in conflict with quantum mechanics and with facts established by experiment." [4].

Many experiments performed reported dramatic violations of Bell's inequality. Most of these results are repeatedly regarded as being inconsistent with the assumption of local realism while consistent with quantum mechanics. But despite of most of these experiments and despite of the different meanings associated with the notion of locality, it is possible to drill very deep into the details of this problem, even into the level of formal mathematical proof. However, in order to avoid misconceptions, the second difficulty is set aside, and a formal definition of the notion locality, entanglement and superposition will not be given.

Bell's theorem and the Chsh inequality are already refuted under some certain circumstances. The aim of this paper is to refute Bell's theorem and the Chsh inequality in general and under any circumstances. The purpose of this present paper is an attempt to recognize the logical structure behind the theorems above and to leave these incorrect theorems logically and definitely behind us. In view of the extremely high precision by which Bell's theorem and the Chsh inequality have been experimentally confirmed, this appears to be not an easy task. In the following, we will provide a mathematical and logical proof that neither Bell's theorem nor the Chsh inequality is logically consistent and mathematically valid. The concept of non-locality in quantum mechanics needs new mathematical foundations.

\section{Material and Methods}

The most important implication of Bell's theorem and one striking aspect of today's quantum mechanics is non locality. Today, this impossibility of a local realistic interpretation of quantum mechanics is back grounded by a family of inequalities known as Bell's theorem or as Chsh inequality. Inequalities as such are very important in many branches of physics and mathematics. In general, an inequality is a relation that holds between two values which are not equal, which are different. In mathematics, analytic number theory often operates with inequalities. Usually, an inequality is denoted by the symbols $<$ or $>$ or by the symbols $\leq$ or $\geq$.

\subsection{Strict Inequalities}

A strict inequality is an inequality where the inequality symbol is either $>$ (strictly greater than) or $<$ (strictly less than). Consequently, a strict inequality is an inequality which has no equality conditions. In terms of algebra, we obtain

$$
a<b
$$

The notation $a<b$ means that " $a$ is strictly less than $b$ ". Equally it is

$$
a>b
$$

The notation $a>b$ means that " $a$ is strictly greater than $b$ ".

\subsection{Non Strict Inequalities}

In contrast to strict inequalities, a non strict inequality is an inequality where the inequality symbol is $\geq$ (either greater than or equal to) or $\leq$ (either less than or equal to). Consequently, a non strict inequality is an inequality which has equality conditions too. In terms of algebra, we obtain

$$
a \leq b
$$

The notation $a \leq b$ means that " $a$ is either less than or equal to $b$ ". Equally it is

$$
a \geq b
$$


The notation $a \geq b$ means that " $a$ is either greater than or equal to $b$ ".

\section{Scholium}

What is the essential of an inequality? Inequalities are governed by many properties. In any case, an inequality must give a true expression. It is not allowed to derive a logical contradiction out of an inequality. Inequalities as such have many properties in common with equalities. As in the case of equations, inequalities can be transformed in several ways and changed even into an equality.

Example

According to Einstein's theory of special relativity, energy and mass are equivalent [5]. In other words it is

$$
o E={ }_{0} m \times c^{2}={ }_{R} E \times \sqrt[2]{1-\frac{v^{2}}{c^{2}}}={ }_{R} m \times c^{2} \times \sqrt[2]{1-\frac{v^{2}}{c^{2}}}
$$

where ${ }_{O} E$ denotes the "rest-energy"; ${ }_{R} E$ denotes the total or "relativistic" energy; $v$ is the relative velocity and $c$ is the speed of light. Consequently, it is possible to claim that (due to special relativity theory) the foundation of special relativity is the non strict inequality ${ }_{R} E \geq{ }_{O} E$. If this non strict inequality is correct it is and must be possible to transfer the same non strict inequality without any contradiction or loss of information into an equality i.e. by adding a term $\Delta E$. Thus far, it is ${ }_{R} E={ }_{O} E+\Delta E$. Clearly, either ${ }_{R} E={ }_{O} E$ or ${ }_{R} E>{ }_{O} E$ but both cannot hold simultaneously, at the same measurement. As soon as $\Delta E=0$ we obtain ${ }_{R} E={ }_{O} E$ and special relativity reduces to simple Newtonian mechanics. Another example is the variance.

As generally known, the variance of ${ }_{R} X_{t}$ is defined as $\sigma\left({ }_{R} X_{t}\right)^{2}=E\left({ }_{R} X_{t}^{2}\right)-E\left({ }_{R} X_{t}\right)^{2}$ where $E\left({ }_{R} X_{t}\right)$ denotes the expectation value of ${ }_{R} X_{t}$. The variance can take different values. In principle, it is possible that $\sigma\left({ }_{R} X_{t}\right)^{2}=0$. The equality $\sigma\left({ }_{R} X_{t}\right)^{2}=E\left({ }_{R} X_{t}^{2}\right)-E\left({ }_{R} X_{t}\right)^{2}$ can be changed into an inequality as $\sigma\left({ }_{R} X_{t}\right)^{2} \geq 0$. This non strict inequality is not the definition of variance but a description of the possible values, the variance can take. Clearly, if an experiment is performed and if it is found that $\sigma\left({ }_{R} X_{t}\right)^{2}=0$ then it is equally found that is not true that $\sigma\left({ }_{R} X_{t}\right)^{2}>0$ and vice versa. If a measurement has determined a varianceof $\sigma\left({ }_{R} X_{t}\right)^{2}>0$, then it is equally found that the variance is not $\sigma\left({ }_{R} X_{t}\right)^{2}=0$. If a variance is equal to zero than the same variance is not greater than zero. If a variance is greater than zero, then the same variance is not equal to zero. Consequently, $\sigma\left({ }_{R} X_{t}\right)^{2} \geq 0$ means that the variance is either greater than or equal to zero but not both in the same respect. In general, an inequality can be transformed into an equally while adding a term $c$ with some special properties and $a \geq b$ changes to $a=b+c$.

\subsection{Einstein and Nonrelativistic Quantum Mechanics}

From the early days of quantum mechanics, Albert Einstein did not hide his dissatisfaction with some principles of the nonrelativistic quantum mechanics. Einstein's dissatisfaction with the nonrelativistic quantum mechanics and especially the great controversy between Einstein and Niels Bohr culminated in a publication entitled as "Can Quantum-Mechanical Description of Physical Reality Be Considered Complete?" [1]. Due to Einstein, Podolsky and Rosen, "the description of reality as given by a wave function is not complete" [6]. Consequently, quantum mechanics is not a complete physical theory but should be supplemented by additional (i.e. local hidden) variables.

\subsection{Einstein and the Principle of Locality}

Einstein's principle of locality is based on the assumption of independence of events. In Dialectica, Einstein wrote:

"Fur die relative Unabhängigkeit räumlich distanter Dinge (A und B) ist die Idee characteristisch: äussere Beeinflussung von A hat keinenunmitte lbaren Einfluss auf B; dies istals “ Prinzip der Nahewirkung” bekannt, das nur in der Feld-Theorie consequent angewendet ist. Völlige Aufhebung dieses Grundsatzes würde die Idee von der Existenz (quasi-) abgeschlossener Systeme und damit die Aufstellung empirisch prüfbarer Gesetze in dem uns geläufigen Sinneunmöglich machen.” [2].

Translated into English:

"The following idea characterizes the relative independence of objects far apart in space (A and B): external influence on A has no direct influence on B; this is known as 'the Principle of Local Action', which is used consistently only in field theory. If this axiom were to be completely abolished, the idea of the existence of quasienclosed systems, and thereby the postulation of laws which can be checked empirically in the accepted sense, would become impossible". 


\subsection{Bell's Inequality/Theorem}

Bell's inequality/theorem has become central to both (quantum) physics and metaphysics since the same touches upon many of the fundamental philosophical issues which relate to modern physics. Following Bell's path of thought, any theory of "causality and locality ... [is, author $].$. incompatible with the statistical predictions of quantum mechanics." [3].

Bell's theorem leaves two options for the nature of reality. Either reality is irreducibly random (no hidden variables which determine the results of individual measurements) or reality is "non-local"" (one measuring device influences another measuring device, however remote). There are several different, mathematical formulations of Bell's inequality/theorem. In 1964 John S. Bell, a native of Northern Ireland, published his theorem in the form of a non strict inequality [7] in the short-lived journal Physics as

$$
1+E(b, c) \geq|E(a, b)-E(a, c)|
$$

where $a, b$ and $c$ are the local detector settings of the apparatus and $E(a, b), E(a, c), E(b, c)$ denote the expectation values. Thus far, the values measured by observers (i.e. Alice or Bob) are only functions of the local detector settings and the hidden parameter. The value observed by Bob with detector setting $b$ is $B(b, \lambda)$, the value observed by Alice with detector setting $a$ is $A(a, \lambda)$. Further, Bell's other vital assumption is that [8]

$$
A(a, \lambda)= \pm 1 \text { and } B(b, \lambda)= \pm 1
$$

Bell's approach to the calculation of quantum mechanical expectation values must ensure the theoretical equivalence of the quantum mechanical expectation values as calculated due to his proposal with the expectation values as calculated due to probability theory and mathematical statistics. Thus far, it is no surprise that there was no difficulty to show, that this is not the case. Using Bell's coding for spin-up and spin-down, incorrect [9] quantum mechanical expectation values will be calculated. From the standpoint of logic and mathematics, Bell's theorem is formulated as a non strict inequality and not as an equality. Besides of all, Bell's theorem possesses all the properties of a non strict inequality and must obey all the needs of a non strict inequality too, whatever the meaning of Bell's theorem may be. In general, Bell's theorem as a non strict inequality is defined something as $A \geq B$. The case $A>B$ is of course different from the case $A=B$. Due to the needs of a non strict inequality these two cases cannot hold both simultaneously. Still Bell's theorem covers both cases and both cases are possible and must be possible in principle. Thus far, following Bell's theorem, we must accept either that

$$
1+E(b, c)=|E(a, b)-E(a, c)|
$$

or that

$$
1+E(b, c)>|E(a, b)-E(a, c)|
$$

but not both simultaneously. In general, under conditions of Equation (9), Bell's theorem demands that

$$
1+E(b, c)-(|E(a, b)-E(a, c)|)>0
$$

\subsection{Bell's Term}

We define Bell's term $\boldsymbol{B}$, which is under conditions of Equation (9) greater than zero, as

$$
B \equiv 1+E(b, c)-(|E(a, b)-E(a, c)|)
$$

\subsection{Chsh Inequality}

Due to Bell's theorem, predictions of quantum mechanics are inconsistent or in disagreement with the assumption of locality. Based on Bell's contribution, Clauser, Horne, Shimony and Holt presented "a generalization of Bell's theorem which applies to realizable experiments" [10] and derived another inequality, the so called Chsh inequality as 


$$
2 \geq\left|E(a, b)-E\left(a^{\prime}, b\right)\right|+\left|E\left(a, b^{\prime}\right)+E\left(a^{\prime}, b^{\prime}\right)\right|
$$

where $a, a^{\prime}, b$ and $b^{\prime}$ are the local detector settings and $E(a, b), E\left(a^{\prime}, b\right), E\left(a, b^{\prime}\right)$ and $E\left(a^{\prime}, b^{\prime}\right)$ denote the expectation values. Meanwhile, there is an extensive literature supporting the validity of the Chsh inequality. In general, the authors of the Chsh inequality are coding spin-up and spin-down, similar to Bell, in a mathematically and logically inconsistent way. “...henceforth interpret $A(a)= \pm 1$ and $B(b)= \pm 1 \ldots$ " [10]. Another far reaching and striking aspect of the Chsh inequality is that the number 2 is defined either as

$$
2=\left|E(a, b)-E\left(a^{\prime}, b\right)\right|+\left|E\left(a, b^{\prime}\right)+E\left(a^{\prime}, b^{\prime}\right)\right|
$$

or equally as

$$
2>\left|E(a, b)-E\left(a^{\prime}, b\right)\right|+\left|E\left(a, b^{\prime}\right)+E\left(a^{\prime}, b^{\prime}\right)\right|
$$

Clauser, Horne, Shimony and Holt are claiming: "Bell's theorem has profound implications in that it points to a decisive experimental test of the entire family of local hidden-variable theories." [11]. One consequence and property of the Chsh inequality under conditions of Equation (14) is that

$$
2-\left(\left|E(a, b)-E\left(a^{\prime}, b\right)\right|+\left|E\left(a, b^{\prime}\right)+E\left(a^{\prime}, b^{\prime}\right)\right|\right)>0
$$

\subsection{Chsh Term}

We define a Chsh term denoted as $C$, which under conditions of Equation (14) is greater than zero, as

$$
C \equiv 2-\left(\left|E(a, b)-E\left(a^{\prime}, b\right)\right|-\left|E\left(a, b^{\prime}\right)+E\left(a^{\prime}, b^{\prime}\right)\right|\right)
$$

\subsection{Axioms}

\section{Axiom I. (Lex identitatis)}

The following theory is based on the axiom:

$$
+1=+1 .
$$

\section{Results}

\subsection{Theorem. Bell's Theorem as An Equality}

Claim

Bell's theorem in the form of an equality demands that

$$
1+E(b, c)=|B>0|+|E(a, b)-E(a, c)|
$$

\section{Direct Proof}

Due to our Axiom I, it is

$$
+1=+1
$$

We add the term $E(b, c)$ and do obtain the relationship

$$
1+E(b, c)=1+E(b, c)
$$

Adding zero or $(|E(a, b)-E(a, c)|)-(|E(a, b)-E(a, c)|)=0$ we obtain

$$
1+E(b, c)=1+E(b, c)-|E(a, b)-E(a, c)|+|E(a, b)-E(a, c)|
$$

Bell's term, denoted as $B$, was defined as $B=1+E(b, c)-(|E(a, b)-E(a, c)|)$. Substituting this term within the equation above, we obtain

$$
1+E(b, c)=B+|E(a, b)-E(a, c)|
$$


Under conditions of Equation (8), Bell's term is equal to zero. Under conditions of Equation (9) and due to Equation (10), Bell's term $B$ is greater than zero or it has to be that $|B>0|$. Due to Equation (9) and Equation (10), Bell's theorem demands equally that

$$
1+E(b, c)=|B>0|+|E(a, b)-E(a, c)|
$$

\section{Quod Erat Demonstrandum.}

\subsection{Theorem. Refutation of Bell's Theorem in General}

\section{Claim}

Bell's theorem is neither logically nor mathematically correct. Thus far, accept Bell's theorem as valid then you must accept too that

$$
+0=+1
$$

\section{Proof by Contradiction}

The technique of a proof by contradiction is widely used in physics, mathematics and philosophy. Thus far, following the principles of a proof by contradiction and in opposite to our claim above, we are sure and claiming that Bell's theorem is mathematically valid and accepting too that Bell's theorem is logically consistent. Consequently, we are not able to derive any logical contradiction out of Bell's theorem. Bell's theorem, accepted as valid and correct, is formulated in the form of a non strict inequality as

$$
1+E(b, c) \geq|E(a, b)-E(a, c)|
$$

Bell's theorem is covering two cases. Bell's theorem is logically and mathematically correct either if

$$
1+E(b, c)=|E(a, b)-E(a, c)|
$$

or if

$$
1+E(b, c)>|E(a, b)-E(a, c)|
$$

Clearly, both implications of Bell's theorem may not hold simultaneously. But this does not make Bell's theorem logically and mathematically inconsistent as such. Besides of all, the term $(1+E(b, c))$ is determined or defined in two different ways, which are both unrestrictedly valid. The following Table 1 is able to illustrate the last relationship.

\section{Table 1. Bell's theorem as a strict inequality.}

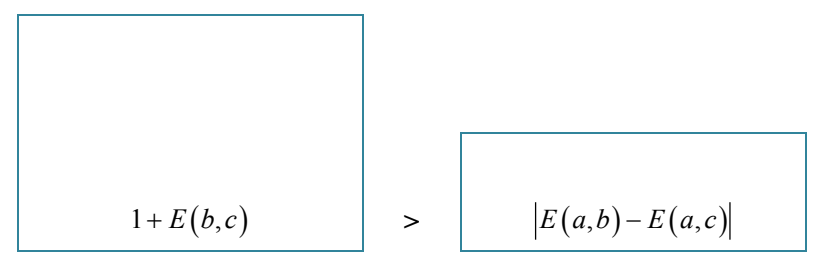

Bell's theorem in the form of a strict inequality must obey the needs of an equality too and this without any contradiction or without any loss of validity. Consequently, since the value of the term $|E(a, b)-E(a, c)|$ is nonnegative, we must accept too, that the term $1+E(b, c)$ is non negative too, since the same is greater than the term $|E(a, b)-E(a, c)|$. Further, theoretically it is possible that the term $|E(a, b)-E(a, c)|$ is equal to zero or $|E(a, b)-E(a, c)|=0$. Inthis case it is $1+E(b, c)>0$. As we have seen above, a strict inequality can be changed into an equality and vice versa. Thus far, let us define a so-called Bell's term (determined as $|B>0|=1+E(b, c)-(|E(a, b)-E(a, c)|))$. Bell's term, denoted as $B$, has some properties. Bell's term transfers Bell's strict inequality into Bell's equality. Thus far, since the term $|E(a, b)-E(a, c)|$ can be equal to zero Bell's term $B$ itself must be greater than zero to ensure Bell's theorem in the form of a strict inequality can be transferred into an equality. Equally, the same term must itself be non negative too. In other words it is $|B>0|$. The following Table 2 may illustrate this relationship. 
Table 2. Bell's theorem as an equality.

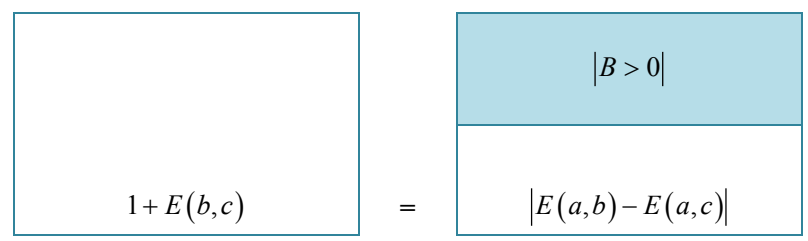

In other words, as already verified, Bell's theorem in the form of a strict inequality, treated as logically and mathematically correct demands according to Equation (9), Equation (10), Equation (11) and Equation (23) that

$$
1+E(b, c)=|E(a, b)-E(a, c)|+|B>0|
$$

In the same respect and due to Equation (8) and Equation (22) Bell's theorem demands equally that

$$
1+E(b, c)=|E(a, b)-E(a, c)|+|B=0|
$$

In general, due to axiom I it is

$$
+1=+1
$$

Adding the term $E(b, c)$ to the Equation (30) we obtain

$$
1+E(b, c)=1+E(b, c)
$$

Following Bell's reasoning, it is the same term $1+E(b, c)$ which is defined in the same respect in two different ways. Thus far, we equate Equation (28) and Equation (29). One finds then straight forwardly that

$$
|E(a, b)-E(a, c)|+|B=0|=|E(a, b)-E(a, c)|+|B>0|
$$

Rearranging Equation (32), we obtain

$$
+|B=0|=+|B>0|
$$

Dividing Equation (33) by $(|B>+0|)$, it is

$$
\frac{+|B=0|}{+|B>0|}=\frac{+|B>0|}{+|B>0|}
$$

or at the end

$$
+0=+1
$$

\section{Quod Erat Demonstrandum}

Thus far, contrary to our starting point and in contrast to our expectation, a logical contradiction can be derived out of Bell's theorem. It is not true that $+0=+1$. Following Bell's reasoning we must accept something, which is obviously incorrect. A logical contradiction is something we try to avoid. In particular, it appears to be very difficult to convince the scientific community that our world is grounded on logical contradictions which are exactly what Bell's theorem demands. Bell's theorem leads to a logical contradiction and is based on a logical contradiction. Consequently, Bell's theorem is refuted in general.

\subsection{Theorem. The Chsh Inequality as an Equality}

\section{Claim}

One consequence of the Chsh Inequality is that

$$
+2=|C>0|+\left(\left|E(a, b)-E\left(a^{\prime}, b\right)\right|-\left|E\left(a, b^{\prime}\right)+E\left(a^{\prime}, b^{\prime}\right)\right|\right)
$$

\section{Direct Proof}

Due to our Axiom I, it is

$$
+1=+1
$$


Multiplying this equation by the number +2 we obtain the relationship

$$
+(1 \times 2)=+(1 \times 2)
$$

Adding zero or $\left(\left|E(a, b)-E\left(a, b^{\prime}\right)\right|+\left|E\left(a^{\prime}, b\right)+E\left(a^{\prime}, b^{\prime}\right)\right|\right)-\left(\left|E(a, b)-E\left(a^{\prime}, b\right)\right|+\left|E\left(a, b^{\prime}\right)+E\left(a^{\prime}, b^{\prime}\right)\right|\right)=0$ we obtain

$$
\begin{aligned}
+2=+2 & \\
& -\left(\left|E(a, b)-E\left(a^{\prime}, b\right)\right|-\left|E\left(a, b^{\prime}\right)+E\left(a^{\prime}, b^{\prime}\right)\right|\right) \\
& +\left(\left|E(a, b)-E\left(a^{\prime}, b\right)\right|-\left|E\left(a, b^{\prime}\right)+E\left(a^{\prime}, b^{\prime}\right)\right|\right)
\end{aligned}
$$

The Chsh term, denoted as $C$, was defined as $C=2-\left(\left|E(a, b)-E\left(a^{\prime}, b\right)\right|+\left|E\left(a, b^{\prime}\right)+E\left(a^{\prime}, b^{\prime}\right)\right|\right)$. Substituting this Chsh term within the equation above, we obtain

$$
+2=C+\left(\left|E(a, b)-E\left(a^{\prime}, b\right)\right|-\left|E\left(a, b^{\prime}\right)+E\left(a^{\prime}, b^{\prime}\right)\right|\right)
$$

Under conditions of Equation (13), the Chsh term $C$ is equal to zero. Under conditions of Equation (14) and due to Equation (15), the Chsh term $C$ is greater than zero or it has to be that $|C>0|$. Due to Equation (14) and Equation (15), the Chsh inequality demands equally that

$$
+2=|C>0|+\left(\left|E(a, b)-E\left(a^{\prime}, b\right)\right|-\left|E\left(a, b^{\prime}\right)+E\left(a^{\prime}, b^{\prime}\right)\right|\right)
$$

\section{Quod erat demonstrandum}

\subsection{Theorem. Refutation of Chsh Inequality}

Under conditions of independence, the Chsh inequality is already refuted [9]. In the following, we will refute the Chsh inequality in general and under any circumstances.

\section{Claim}

The Chsh inequality is logically and mathematically incorrect. If you accept the Chsh inequality as generally valid then you must accept too that

$$
+0=+1
$$

\section{Proof by contradiction}

In general, the Chsh inequality is formulated as [12]

$$
2 \geq\left|E(a, b)-E\left(a^{\prime}, b\right)\right|+\left|E\left(a, b^{\prime}\right)+E\left(a^{\prime}, b^{\prime}\right)\right|
$$

As a non strict inequality, the Chsh inequality is determined by an equality or by the claim that

$$
2=\left|E(a, b)-E\left(a^{\prime}, b\right)\right|+\left|E\left(a, b^{\prime}\right)+E\left(a^{\prime}, b^{\prime}\right)\right|
$$

and by a strict inequality or by the claim that

$$
2>\left|E(a, b)-E\left(a^{\prime}, b\right)\right|+\left|E\left(a, b^{\prime}\right)+E\left(a^{\prime}, b^{\prime}\right)\right|
$$

Due to the Chsh inequality, both parts of the non strict Chsh inequality, the equality and the strict inequality, must be correct and valid in principle. Otherwise the non strict Chsh inequality would be incorrect from the beginning. Formally, both forms of the CHSH inequality are defining the number 2 in two different ways. The strict form of the Chsh inequality can be illustrated as follows (Table 3 ).

Table 3. The strict form of the Chsh inequality.

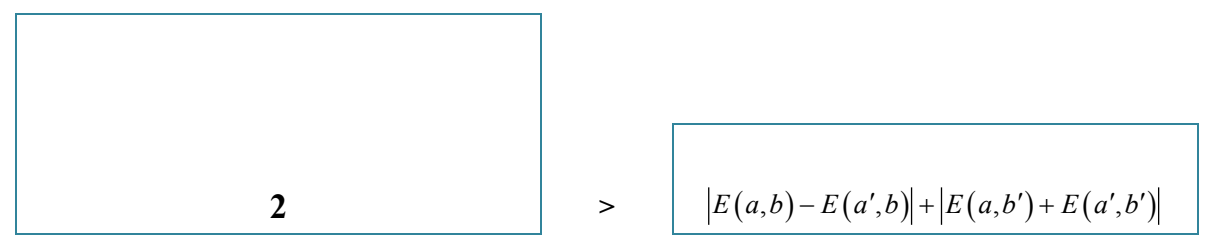


It is possible to convert a strict form of an inequality into an equality and vice versa. To proceed further, we can transfer the strict from of the Chsh inequality into an equality by adding a special term, the Chsh term $\left(C=2-\left(\left|E(a, b)-E\left(a^{\prime}, b\right)\right|+\left|E\left(a, b^{\prime}\right)+E\left(a^{\prime}, b^{\prime}\right)\right|\right)\right)$ denoted as $C$, which under conditions of Equation (14) must be greater than zero or $+C>+0$. The following picture illustrates this far reaching relationship once again to achieve another point of view (Table 4).

Table 4. The strict form of the Chsh inequality.

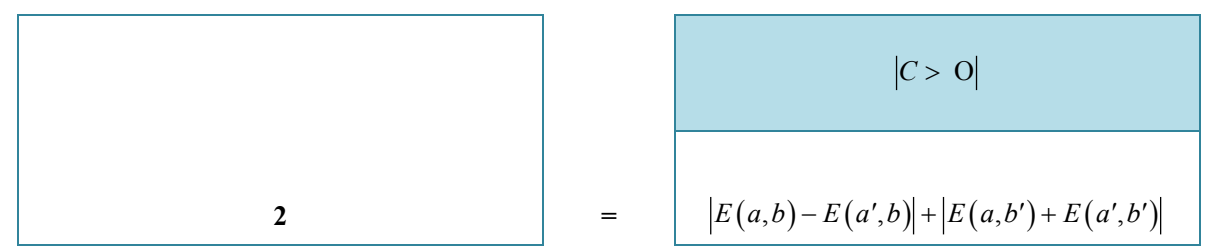

In other words, the Chsh inequality may be written as "the superposition of two terms". As already verified, the Chsh inequality, treated as logically and mathematically correct demands according to Equation (14), Equation (15), Equation (16) and Equation (41) that

$$
+2=|C>0|+\left(\left|E(a, b)-E\left(a^{\prime}, b\right)\right|-\left|E\left(a, b^{\prime}\right)+E\left(a^{\prime}, b^{\prime}\right)\right|\right)
$$

and according to Equation (13), Equation (16) and Equation (40) that

$$
+2=+|C=0|+\left|E(a, b)-E\left(a^{\prime}, b\right)\right|+\left|E\left(a, b^{\prime}\right)+E\left(a^{\prime}, b^{\prime}\right)\right|
$$

In general, due to axiom I it is

$$
+1=+1
$$

Multiplying Equation (48) by the number +2 we obtain

$$
+2=+2
$$

Substituting Equation (47) into this equation we obtain

$$
\left|E(a, b)-E\left(a^{\prime}, b\right)\right|+\left|E\left(a, b^{\prime}\right)+E\left(a^{\prime}, b^{\prime}\right)\right|=+2
$$

Substituting Equation (46) into this equation we obtain

$$
\begin{aligned}
& \left|E(a, b)-E\left(a^{\prime}, b\right)\right|+\left|E\left(a, b^{\prime}\right)+E\left(a^{\prime}, b^{\prime}\right)\right| \\
& =|C>0|+\left|E(a, b)-E\left(a^{\prime}, b\right)\right|+\left|E\left(a, b^{\prime}\right)+E\left(a^{\prime}, b^{\prime}\right)\right|
\end{aligned}
$$

Subtracting $\left|E(a, b)-E\left(a^{\prime}, b\right)\right|+\left|E\left(a, b^{\prime}\right)+E\left(a^{\prime}, b^{\prime}\right)\right|$ and collecting together the terms, we obtain

$$
+|C=0|=+|C>0|
$$

Dividing Equation (52) by the term $|C>0|$ we must accept too that

$$
\frac{+|C=0|}{+|C>0|}=\frac{+|C>0|}{+|C>0|}
$$

or at the end that

$$
+0=+1
$$

\section{Quod erat demonstrandum}

In other words, as already verified by our direct proof before, the Chsh inequality leads to a logical contradiction and is based on a logical contradiction. The Chsh inequality, the general form of Bell's theorem, is refuted in general. 


\section{Discussion}

The results of this publication are based on the law of non-contradiction (LNC) as one of the foremost among the principles of science. In particular, in our today's understanding of the foundations of science as such, logical contradictions cannot be accepted as the foundation of science or of our thinking.

In other words, is it logically and mathematically justified to claim something like $+1 \geq+0$. If yes, we must accept the possibility that $+1>+0$. This part of the non strict inequality $+1 \geq+0$ is in accordance with our today's understanding of science as such. But the non-strict inequality $+1 \geq+0$ implies the validity and the logical consistency of another claim too. Due to the non strict inequality $+1 \geq+0$ the claim $+1=+0$ must be valid too. It is not necessary that both claims are valid simultaneously, but both claims must be valid in principle. The last claim that $+1=+0$ is neither logically nor mathematically consistent. Thus far, we are not allowed to claim that $+1 \geq+0$, especially it is difficult to convince the scientific community that $+1=+0$. Scientist and philosophers have to draw a very sharp line between correct and not correct to assure that logical contradictions are avoided.

Some other experimentalists and theoreticians accused Bell's theorem and the Chsh inequality of being incorrect but without a definite and general refutation in sight. Most recently, Bell's theorem and the Chsh inequality were refuted [9] under some certain conditions. When we tried to determine whether Bell's theorem and the Chsh inequality are logically consistent and mathematically correct, we were able to derive a logical contradiction. In contrast to our expectation, Bell's theorem and the Chsh inequality contain some serious mathematical and physical deficiencies which leads to a logical contradiction and which proofs them as invalid. In other words, a seemingly sound piece of reasoning (i.e. Bell's theorem or the Chsh inequality) based on apparently true assumptions cannot lead to analogical contradictions. Bell's theorem and the Chsh inequality are refuted in general. According to the Copenhagen dominated a causal interpretation of quantum mechanics, the principle of causality occupied an important place in the history of the philosophical interpretation of quantum mechanics from the beginning. It was Heisenberg himself who claimed that his uncertainty principle has refuted the principle of causality. Due to Heisenberg: “...so wirddurch die Quantenmechanik die Ungültigkeit des Kausalgesetzes definitiv festgestellt" [13]. Bohr, another 'founding father' of the Copenhagen dominated acausal interpretation of quantum mechanics, in his striving to find a common ground for our knowledge, addressed the assembly of scientists by demanding that "physics ... forces us to replace ... causality by ... complementarity" [14]. Meanwhile, Heisenberg's uncertainty principle [13] together with the several different, mathematical re-formulations of the same like Ozawa's "universally valid uncertainty relation" is proofed as not correct [15] and thus far no longer valid [16]. Finally, some basic pillars of today's a causal Copenhagen dominated interpretation of quantum mechanics are describing fundamental quantum-mechanical phenomena in a logically inconsistent way. Consequently, a new and unique principle as the starting point for the unification of quantum and relativity theory appears to be necessary. In contrast to the Copenhagen dominated a causal interpretation of quantum mechanics, the principle of causality [17], valid and correct since thousands of years, can be such a principle and is a common ground for our knowledge.

\section{Conclusion}

Bell's theorem and the Chsh inequality are refuted in general.

\section{References}

[1] Einstein, A., Podolsky, B. and Rosen, N. (1935) Can Quantum-Mechanical Description of Physical Reality Be Considered Complete? Physical Review, 47, 777-780. http://dx.doi.org/10.1103/PhysRev.47.777

[2] Einstein, A. (1948) Quanten-Mechanik und Wirklichkeit. Dialectica, 2, 320-324. http://dx.doi.org/10.1111/j.1746-8361.1948.tb00704.x

[3] Bell, J.S. (1964) On the Einstein Podolsky Rosen Paradox. Physics, 1, 195.

[4] d'Espagnat, B. (1979) The Quantum Theory and Reality. Scientific American, 241, 158.

[5] Einstein, A. (1918) "Da Masse und Energie nach den Ergebnissen der speziellen Relativitäts theorie das Gleiche sind..." Prinzipielleszur Allgemeinen Relativitätstheorie. Annalen der Physik, 55, 241-242.

[6] Einstein, A., Podolsky, B. and Rosen, N. (1935) Can Quantum-Mechanical Description of Physical Reality Be Considered Complete? Physical Review, 47, 777. http://dx.doi.org/10.1103/PhysRev.47.777

[7] Bell, J.S. (1964) On the Einstein Podolsky Rosen Paradox. Physics, 1, 198. 
[8] Bell, J.S. (1964) On the Einstein Podolsky Rosen Paradox. Physics, 1, 196.

[9] Barukčić, I. (2012) Anti-Bell-Refutation of Bell's Theorem. In: Atmanspacher, H., Bengtsson, I., Brandenburger, A., Fuchs, C., Haven, E., Hosoya, A., Khrennikov, A., Ozawa, M., Stenholm, S. and Tollaksen, J., Eds., AIP Conference Proceedings: QTRF6 - Quantum Theory: Reconsideration of Foundations, Växjö, 11-14 June 2012, 354-358.

[10] Clauser, J.F., Horne, M.A., Shimony, A. and Holt, R.A. (1969) Proposed Experiment to Test Local Hidden-Variable Theories. Physical Review Letters, 23, 881 .

[11] Clauser, J.F., Horne, M.A., Shimony, A. and Holt, R.A. (1969) Proposed Experiment to Test Local Hidden-Variable Theories. Physical Review Letters, 23, 880. http://dx.doi.org/10.1103/PhysRevLett.23.880

[12] Weihs, G., Jennewein, T., Simon, C., Weinfurter, H. and Zeilinger, A. (1998) Violation of Bell's Inequality under Strict Einstein Locality Conditions. Physical Review Letters, 81, 5039-5043. http://dx.doi.org/10.1103/PhysRevLett.81.5039

[13] Heisenberg, W. (1927) Über den anschaulichen Inhalt der quantentheoretischen Kinematik und Mechanik. Zeitschrift für Physik, 43, 197. http://dx.doi.org/10.1007/BF01397280

[14] Bohr, N. (1937) Causality and Complementarity. Philosophy of Science, 4, 290-291. http://dx.doi.org/10.1086/286465

[15] Barukčić, I. (2011) Anti Heisenberg-Refutation of Heisenberg's Uncertainty Relation. American Institute of Physics: Conference Proceedings, 1327, 322-325. http://dx.doi.org/10.1063/1.3567453

[16] Barukčić, I. (2014) Anti Heisenberg-Refutation of Heisenberg's Uncertainty Principle. International Journal of Applied PhysicsandMathematics, 4, 244-250. http://dx.doi.org/10.7763/IJAPM.2014.V4.292

[17] Barukčić, I. (2016) The Mathematical Formula of the Causal Relationship K. International Journal of Applied Physicsand Mathematics. (In Print) 